Article

\title{
Epitaxial Growth of GaN on Magnetron Sputtered AlN/Hexagonal BN/Sapphire Substrates
}

\author{
Jinxing $\mathrm{Wu}^{1}{ }^{1} \mathbb{B}$, Peixian $\mathrm{Li}^{1}$, Shengrui $\mathrm{Xu}^{2, *}$, Xiaowei Zhou ${ }^{1, *}$, Hongchang Tao ${ }^{2}$, Wenkai Yue ${ }^{1}{ }^{\circledR}$, \\ Yanli Wang ${ }^{1}{ }^{\mathbb{D}}$, Jiangtao $\mathrm{Wu}^{2}$, Yachao Zhang ${ }^{2}$ and Yue Hao ${ }^{2}$ \\ 1 Wide Bandgap Semiconductor Technology Disciplines State Key Laboratory, School of Advanced Materials \\ and Nanotechnology, Xidian University, Xi'an 710071, China; jinxing_wu_xidian@163.com (J.W.); \\ pxli@mail.xidian.edu.cn (P.L.); yuewenkai888@gmail.com (W.Y.); ylwang055065@163.com (Y.W.) \\ 2 Wide Bandgap Semiconductor Technology Disciplines State Key Laboratory, School of Microelectronics, \\ Xidian University, Xi'an 710071, China; hchtao@stu.xidian.edu.cn (H.T.); aweaomeivan@gmail.com (J.W.); \\ ychzhang@xidian.edu.cn (Y.Z.); yhao@xidian.edu.cn (Y.H.) \\ * Correspondence: srxu@xidian.edu.cn (S.X.); xwzhou@mail.xidian.edu.cn (X.Z.); Tel.: +86-138-0919-7527 (S.X.); \\ $+86-180-9266-1260$ (X.Z.)
}

Received: 16 October 2020; Accepted: 11 November 2020; Published: 13 November 2020

\begin{abstract}
Magnetron sputtering is adopted to deposit 25 nm thick AlN on the surface of hexagonal $\mathrm{BN}(\mathrm{h}-\mathrm{BN}) /$ sapphire substrates, followed by epitaxial GaN growth on top of the AlN/h-BN/sapphire substrate using a metal-organic chemical vapor deposition system. Compared to GaN grown on the h-BN/sapphire surface directly, this method results in a continuous and smooth GaN film with a smaller root mean square roughness. Besides, the introduction of the sputtered AIN layer reduces the dislocation density of GaN by $35.7 \%$. We provide a pathway of GaN epitaxy on the h-BN surface, which significantly improves its surface morphology and crystal quality.
\end{abstract}

Keywords: h-BN; sapphire; magnetron sputtered AlN; GaN

\section{Introduction}

Devices manufactured on III-nitride materials are widely being applied in the military, industry, and our daily lives [1-4]. GaN-based light-emitting diodes (LEDs) are used in lighting, backlighting, and decoration [5,6]. Additionally, GaN-based high electron mobility transistors (HEMTs) are adopted in radio frequency and power devices [7]. To obtain III-nitride materials, substrates including sapphire, silicon, and $\mathrm{SiC}$ are mostly utilized. The III-nitride material forms a strong covalent $s p^{3}$ bond with the substrate, and single-crystal GaN cannot be grown on a polycrystalline or amorphous substrate [8]. However, once the epitaxial layer can be released from the substrate, it is a promising solution to the limitations of the substrate. Traditional techniques including laser lift-off and chemical methods must break the covalent bond between the III-nitride material and the substrate, thus causing material damage, and the process is also time-consuming $[9,10]$.

The van der Waals epitaxy of III-nitrides on two-dimensional materials has been a hot research topic in recent years [11-19]. The two-dimensional material h-BN serves as an ideal layer for mechanically releasing devices from the substrate [20]. Since there are no dangling bonds on the surface of the two-dimensional material h-BN or graphene, III-nitrides will form clusters on the surface [21]. It is rather hard to grow III-nitride materials directly on the surface of two-dimensional materials [22]. To overcome this problem, some approaches have been proposed. Wu et al. treated h-BN with O plasma to produce dangling bonds on its surface [23]. The dangling bonds on the h-BN surface facilitate the nucleation of the AIN epitaxial layer. Based on this method, UV-LED devices with an emission wavelength of $\sim 281 \mathrm{~nm}$ were successfully achieved [24]. Graphene treated with N plasma 
introduces dangling bonds on its surface. Dangling bonds are more conducive to the nucleation of AlN. AlN nucleation points continue to grow to form small islands through epitaxy, and then the small islands continue to merge and eventually form a smooth surface [25]. To date, the main method of III-nitride epitaxy on the surface of two-dimensional materials is to generate dangling bonds through plasma treatment, followed by epitaxially growing III-nitride materials. This method results in covalent bond formation between III-nitrides and two-dimensional materials, while it is not fully van der Waals epitaxy.

As previously reported, the improvement of the crystal quality of heteroepitaxial GaN film through the introduction of the AlN buffer layer was widely investigated [26-28]. However, there are few studies focused on how to improve the crystal quality of GaN on two-dimensional materials. In this work, we propose a pathway for the GaN epitaxy based on h-BN/sapphire substrates. A $25 \mathrm{~nm}$ thick AlN layer is deposited on the h-BN/sapphire substrate by magnetron sputtering, followed by the epitaxy process via a metal-organic chemical vapor deposition (MOCVD) system. After introducing the sputtered AlN layer, the surface morphology of GaN is greatly improved, and the crystal quality is promoted. Compared to the plasma treatment of h-BN, this method avoids the damage of $\mathrm{h}-\mathrm{BN}$.

\section{Experiment}

To obtain the h-BN/sapphire substrate, a few layers of h-BN are transferred from the copper foil to the $c$-plane sapphire substrate. The few layers of h-BN are commercial products (SixcarbonTech, Shenzhen, China), here, they are made on a $\mathrm{Cu}$ foil by low-pressure chemical vapor deposition. As shown in Figure 1, based on the h-BN/sapphire substrate, we grow $6 \mu \mathrm{m}$ thick GaN films for sample A. Meanwhile, for sample B, the $25 \mathrm{~nm}$ thick AlN layer is sputtered through magnetron sputtering before GaN growth, and then $6 \mu \mathrm{m}$ thick GaN is grown.

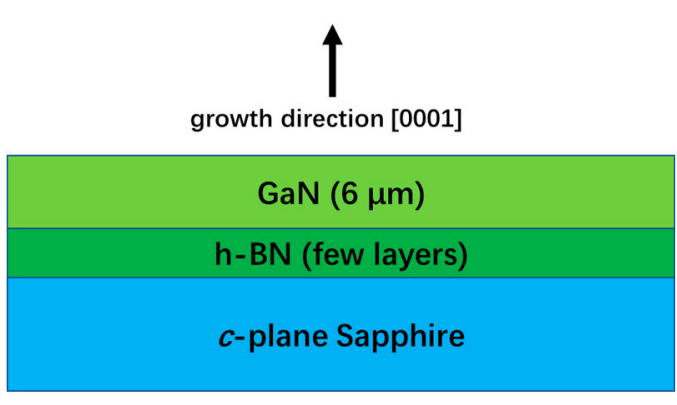

(a) Sample A

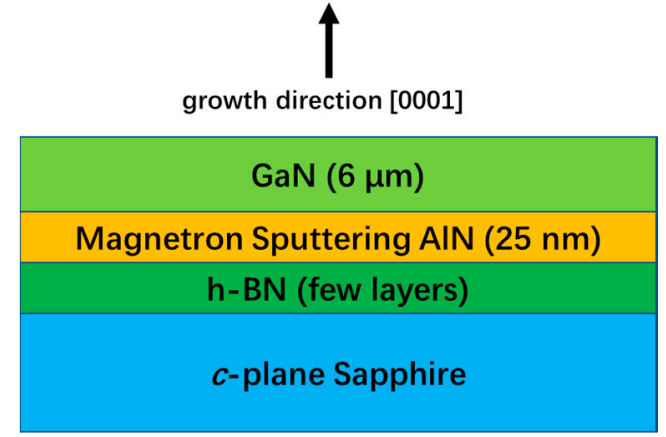

(b) Sample B

Figure 1. (a) GaN material epitaxially grown on h-BN/sapphire substrate of sample A and (b) GaN material epitaxially grow on h-BN/sapphire substrate of sample B after magnetron sputtering $25 \mathrm{~nm}$ thick AlN.

The AIXTRON CRIUS II MOCVD system [29] (Herzogenrath, Germany) is utilized for GaN epitaxial growth. The $25 \mathrm{~nm}$ thick nucleation layer is grown at $520^{\circ} \mathrm{C}$, in which the flow rate of $\mathrm{NH}_{3}$ is $12,500 \mathrm{~mL} / \mathrm{min}$, and the flow rate of TMGa is $75 \mathrm{~mL} / \mathrm{min}$. The pressure during the growth of the nucleation layer is $600 \mathrm{mbar}$. After that, the temperature is raised to $965^{\circ} \mathrm{C}$ to start the growth of the $3 \mathrm{D}$ layer, the flow rate of $\mathrm{NH}_{3}$ is $24,000 \mathrm{~mL} / \mathrm{min}$, the flow rate of $\mathrm{TMGa}$ is $210 \mathrm{~mL} / \mathrm{min}$, the pressure is $300 \mathrm{mbar}$, and the total thickness is about $350 \mathrm{~nm}$. Finally, the temperature is raised to $1080{ }^{\circ} \mathrm{C}$ for $2 \mathrm{D}$ layer growth, the flow rate of $\mathrm{NH}_{3}$ is $28,000 \mathrm{~mL} / \mathrm{min}$, the flow rate of TMGa is $440 \mathrm{~mL} / \mathrm{min}$, the pressure is $300 \mathrm{mbar}$, and the growth thickness is about $5.625 \mu \mathrm{m}$. ITOPS A320 equipment of North Huachuang (Beijing, China) is used for magnetron sputtering. For sample B, the Al target is bombarded with Ar at $600{ }^{\circ} \mathrm{C}$, and $\mathrm{Al}$ ions are sputtered and combine with $\mathrm{N}$ ions to generate $\mathrm{AlN}$, which is then deposited on the surface of the h-BN/sapphire substrate. In order to systematically investigate the samples, various measurements are performed. Scanning electron microscope (SEM) images are taken with a 
FEI MLA650F system (Hillsboro, OR, USA) at an accelerated voltage of $25 \mathrm{kV}$, and the magnification is $8288 \times$. Transmission electron microscopy (TEM) investigations are performed with a Talos F200X (Thermo Fisher, Waltham, MA, USA), operated at $200 \mathrm{kV}$. The detailed surface morphology of the samples is further characterized by an atomic force microscope (AFM) under tapping mode in a Bruker ICON Dimension system (Billerica, MA, USA). Optical properties are obtained by a photoluminescence (PL) spectrometer, 2-679HR-HR 800 (Horiba, Palazzo, France), with a He-Cd laser operating at $325 \mathrm{~nm}$. Additionally, the Raman spectra are achieved by Jobin Yvon LanRam HR800 (Horiba, Palazzo, France) with the excitation wavelength at $514 \mathrm{~nm}$. X-ray diffraction measurements are performed in an $X^{\prime}$ Pert PRO (PANalytical, Almelo, Holland) system using the $\mathrm{Cu}-\mathrm{K}$ line system with a wavelength at $0.154 \mathrm{~nm}$. Finally, we obtain the X-ray photoelectron spectrometer (XPS) results with a ESCALAB 250Xi (Thermo Fisher, Waltham, MA, USA) system using Al-K $\alpha$ radiation $(\lambda=1486.6 \mathrm{eV})$.

\section{Results and Discussion}

Both samples A and B are investigated by SEM to observe the surface topography of the GaN film. As shown in Figure 2a, there are many pits of different sizes in sample A. Since there are no dangling bonds on the surface of $\mathrm{h}-\mathrm{BN}, \mathrm{GaN}$ is difficult to nucleate on its surface. The low nucleation density results in sparse GaN islands and the formation of a smooth and continuous GaN film is difficult or even not possible [22]. As shown in Figure 2b, after $25 \mathrm{~nm}$ thick AlN on the surface of $\mathrm{h}-\mathrm{BN}$ is sputtered, the problem of GaN nucleation on the surface of $\mathrm{h}-\mathrm{BN}$ is alleviated. A smooth and continuous GaN film is obtained for sample B.
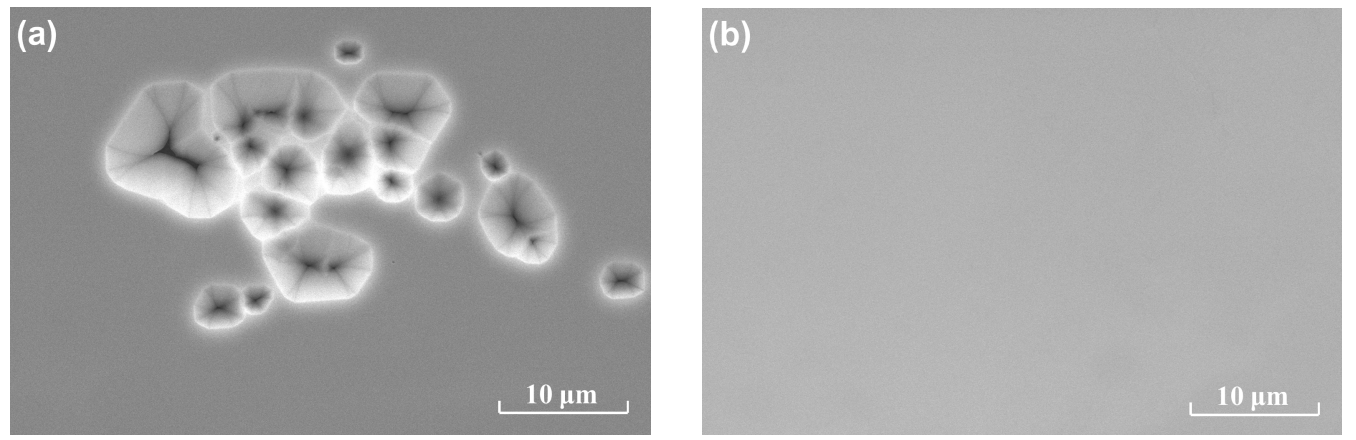

Figure 2. SEM images of (a) sample A and (b) sample B.

To further investigate the two samples, Figure 3a,b show high-resolution TEM cross-sectional images of samples A and B, respectively. We can clearly observe the few layers of h-BN with a thickness of approximately $4 \mathrm{~nm}$ located on the sapphire substrate. In Figure 3a, the upper layer of h-BN is $\mathrm{GaN}$. In Figure 3b, above the few layers of h-BN is $25 \mathrm{~nm}$ thick AlN, and $\mathrm{GaN}$ is above the AlN layer. Figure $3 c, d$ are the EDS diagrams of $\mathrm{Al}$ and $\mathrm{N}$. There is AlN about $25 \mathrm{~nm}$ thick on the h-BN/sapphire substrate, which proves the existence of $25 \mathrm{~nm}$ sputtered AlN in sample B.

Figure 4 shows the AFM images of the two samples within $5 \mu \mathrm{m} \times 5 \mu \mathrm{m}$. Figure $4 \mathrm{a}$ is the AFM image of sample $A$, and the root mean square (RMS) roughness is $1.25 \mathrm{~nm}$, while the RMS roughness of sample B is $0.474 \mathrm{~nm}$. Obviously, sample B has less RMS roughness and a smoother surface, which is consistent with the SEM results shown in Figure 2. 

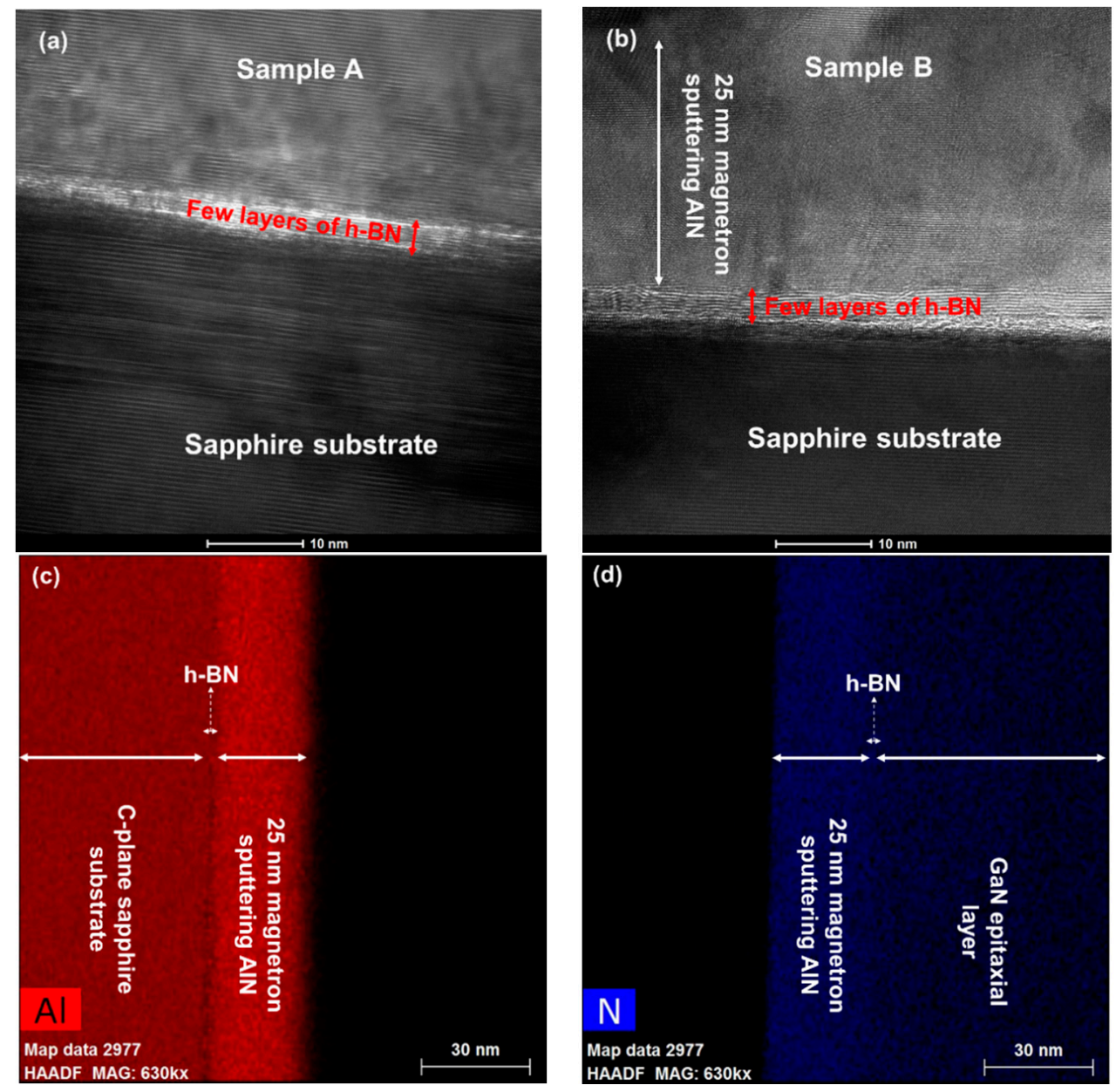

Figure 3. High-resolution TEM cross-sectional view of (a) sample A and (b) sample B. Energy dispersive spectroscopy (EDS) diagrams of (c) Al element and (d) $\mathrm{N}$ element.
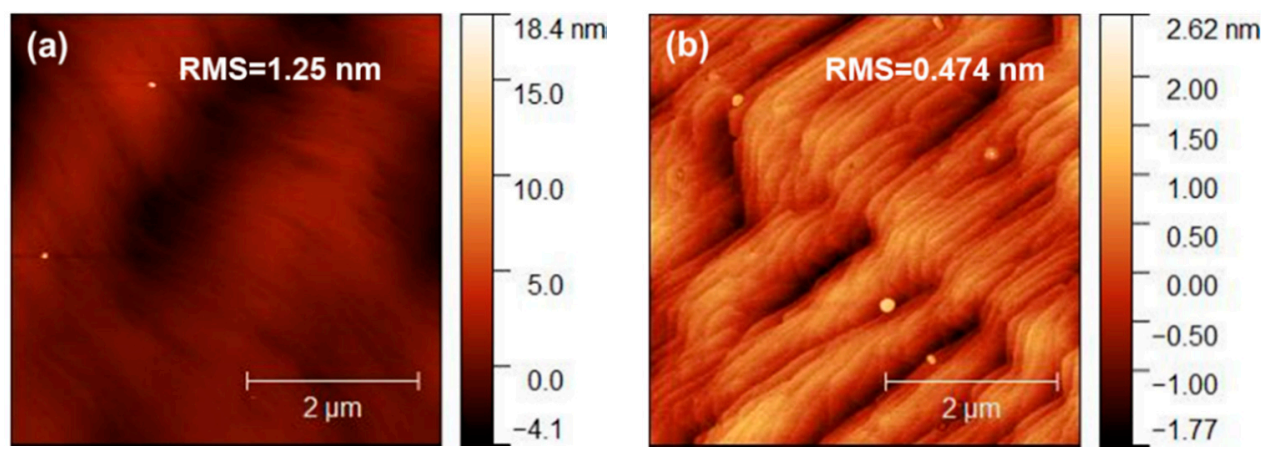

Figure 4. The $5 \mu \mathrm{m} \times 5 \mu \mathrm{m}$ AFM images of (a) sample A and (b) sample B.

As shown in Figure 5, we further investigated the PL and Raman properties of the two samples. Figure 5 a shows that the near band energy emission peaks of the two samples are both at $362.7 \mathrm{~nm}$. The PL intensity of sample B is three times that of sample A, which indicates the improved crystal quality of sample B [30]. Figure $5 \mathrm{~b}$ presents the Raman spectra of the two samples, in which the peak of the sapphire is located at $418.8 \mathrm{~cm}^{-1}$ and the $E_{2}$ (high) peak of the two samples is located at $569.7 \mathrm{~cm}^{-1}$. The vibration mode of $E_{2}$ (high) is most sensitive to stress, and the peak of $E_{2}$ (high) for unstrained $\mathrm{GaN}$ is located at $567.6 \mathrm{~cm}^{-1}$ [31]. The $\mathrm{E}_{2}$ (high) peaks of both samples are located on the right side of $567.6 \mathrm{~cm}^{-1}$, indicating both samples are under a compressive stress state [32]. Besides, the intensity of 
the $E_{2}$ (high) peak of sample $B$ is 2.1 times higher than that of sample A, so the crystal quality of sample $\mathrm{B}$ is improved [33]. According to the results of PL and Raman measurements, the optical properties of sample B are better than sample A.
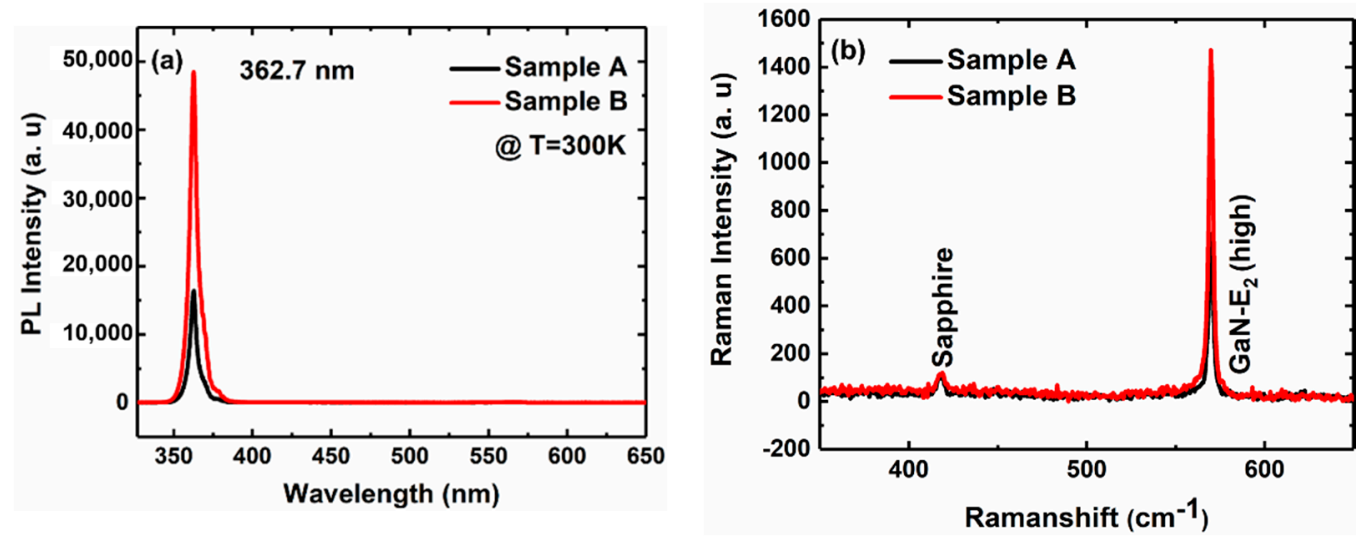

Figure 5. (a) PL and (b) Raman spectra of samples A and B at room temperature (300 K).

To further evaluate the crystal quality of the two samples, we performed an X-ray rocking curve (XRC) measurement. As shown in Figure 6, the (0002)/(10-12) XRC full width at half maxima (FWHM) of samples A and B are 525/501 arcsec and 486/420 arcsec, respectively. The total dislocation density of sample A is $1.9 \times 10^{9} \mathrm{~cm}^{-2}$, while sample B is $1.4 \times 10^{9} \mathrm{~cm}^{-2}$, calculated from XRC results, which is $35.7 \%$ lower than that of sample A [34]. Therefore, sample B features better crystal quality [35], which is consistent with the optical measurements shown in Figure 5.
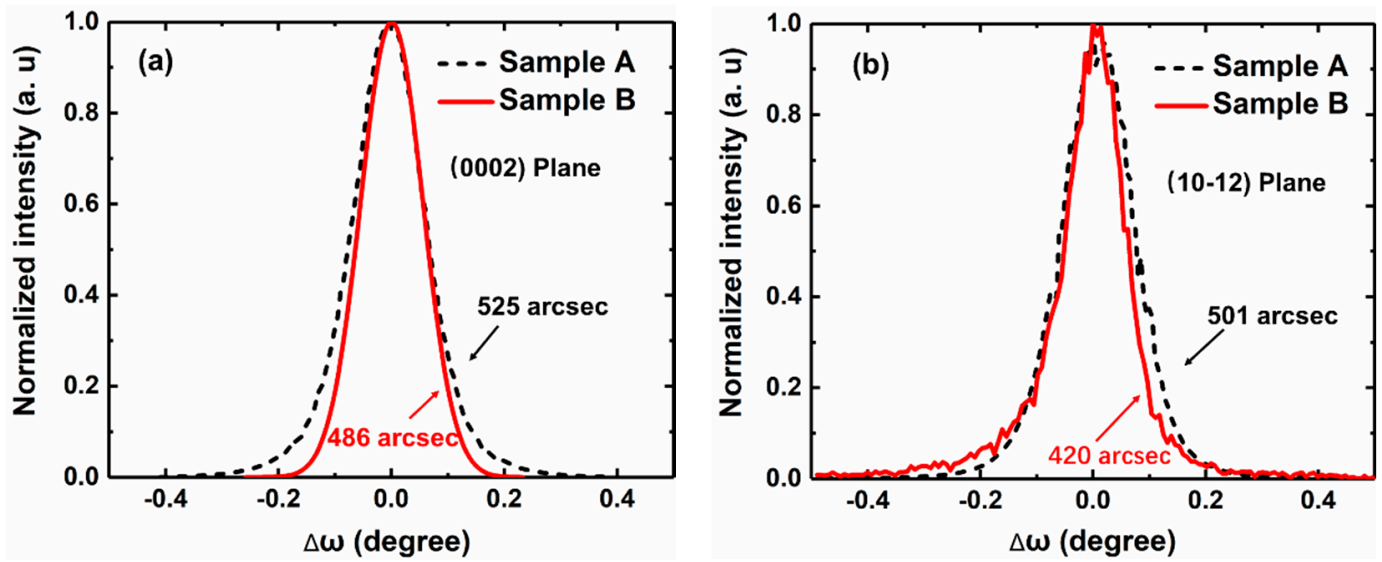

Figure 6. X-ray rocking curves of samples A and B on (a) (0002) and (b) (10-12) reflections.

Figure 7 is the XPS results of the h-BN/sapphire substrate. The B 1s peak and $\mathrm{N}$ 1s peak can be seen from the figure, and the presence of $h-B N$ can be confirmed. The Al $2 p$ peak and O 1s peak confirm the presence of the sapphire substrate. The $C 1$ s peak comes from poly (methyl methacrylate) produced during the transfer of $\mathrm{h}-\mathrm{BN}$ from copper foil to sapphire. 


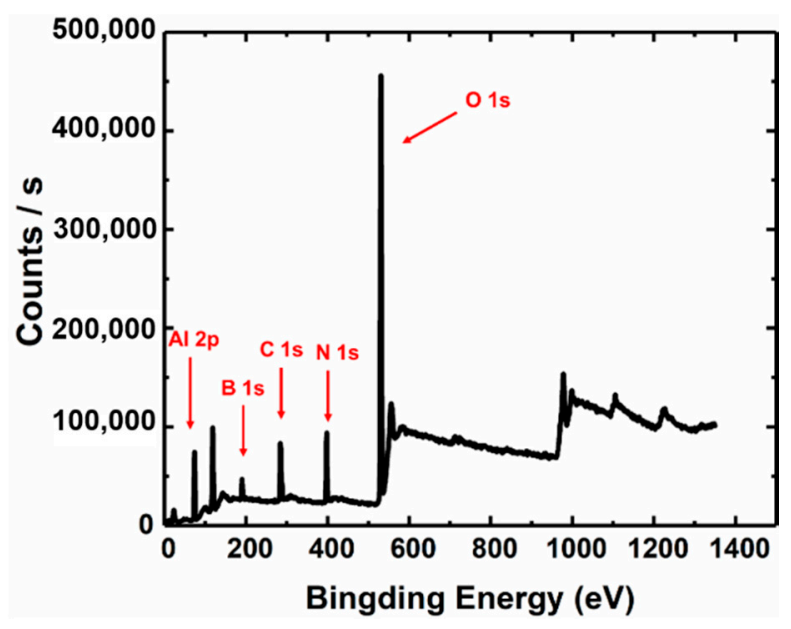

Figure 7. XPS results of h-BN/sapphire substrate.

Based on the model of Wu et al. [23], we further explained the growth mechanism of samples A and B. Figure 8 shows the epitaxial mechanism of GaN on the h-BN/sapphire substrate and sputtered AlN/h-BN/sapphire substrate. Figure 8 a shows the nucleation process of low-temperature GaN on the h-BN/sapphire substrate. Since there are no dangling bonds on the h-BN surface, the nucleation points are sparse and random. Figure $8 \mathrm{~d}$ shows the nucleation process of low-temperature $\mathrm{GaN}$ on the sputtered AlN/h-BN/sapphire substrate. Due to the presence of the sputtered AlN buffer layer, $\mathrm{GaN}$ nucleation sites are dense. As the temperature increases, $\mathrm{GaN}$ nucleation islands gradually grow. Figure $8 \mathrm{~b}$,e show the $\mathrm{GaN}$ islands on the two substrates. Since $\mathrm{GaN}$ has denser nucleation points on the sputtered AlN/h-BN/sapphire, the number of GaN islands is also greater. Finally, the GaN islands merged and the two-dimensional growth begins. Figure 8c,f show the final GaN film. GaN does not form a smooth continuous film on the surface of the h-BN/sapphire substrate. There are many pits of different sizes on the GaN surface. In contrast, GaN epitaxially grown on sputtered AlN/h-BN/sapphire substrate has a continuous smooth film.

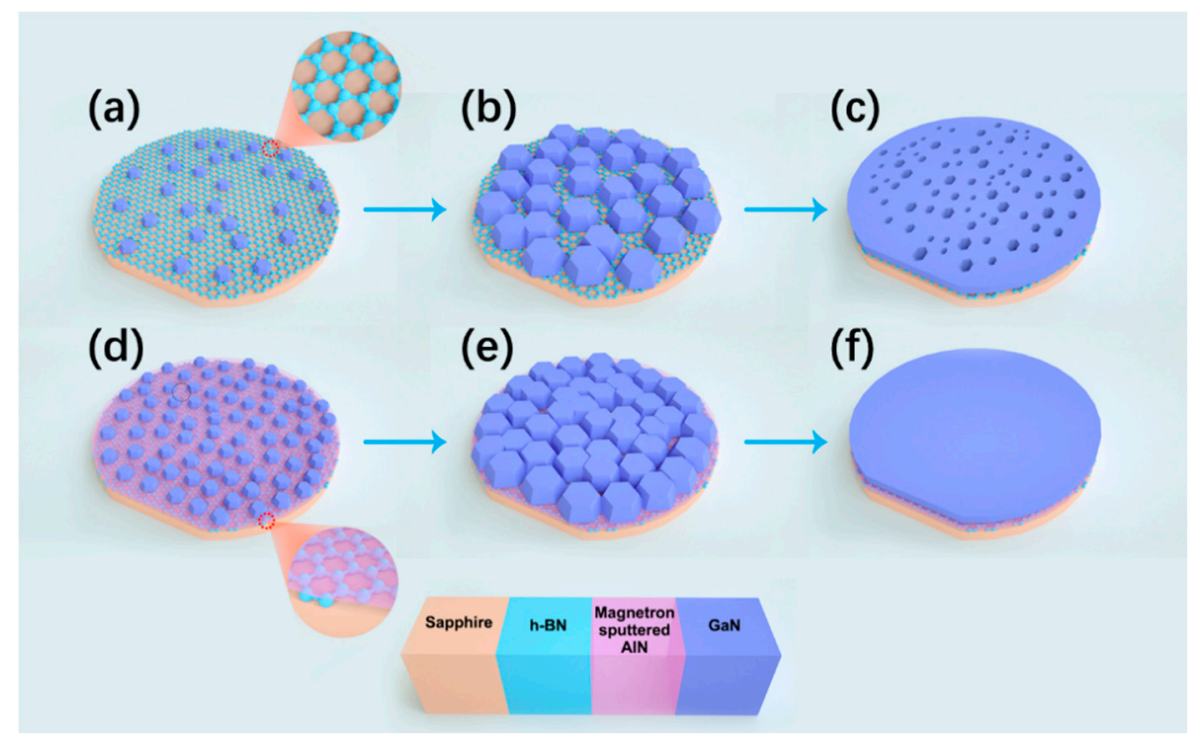

Figure 8. The epitaxial mechanism of $\mathrm{GaN}$ on $\mathrm{h}-\mathrm{BN} / \mathrm{sapphire}$ substrate and sputtered AlN/h-BN/sapphire substrate: (a) GaN nucleation process, (b) formation of GaN islands and (c) GaN film on h-BN/sapphire substrate; (d) GaN nucleation process, (e) formation of GaN islands and (f) GaN film on sputtered AlN/h-BN/sapphire substrate. 


\section{Conclusions}

In summary, due to the lack of dangling bonds on the surface of h-BN, the direct growth of GaN on its surface will make it difficult for GaN to form a smooth and continuous film. We have proposed a pathway for epitaxial GaN on h-BN/sapphire substrates. A $25 \mathrm{~nm}$ thick AlN layer is deposited on top of the h-BN by magnetron sputtering, followed by the epitaxial growth of GaN via MOCVD. As a result, it is verified by SEM that the GaN surface is smooth, and the RMS roughness of the $5 \mu \mathrm{m} \times 5 \mu \mathrm{m}$ AFM image is only $0.474 \mathrm{~nm}$. According to the test results of PL and Raman spectra, the GaN epitaxy based on sputtered AlN/h-BN/sapphire substrate has better crystal quality, which is consistent with the results of XRCs. Finally, we explained the mechanism of sputtered AlN/h-BN/sapphire substrate epitaxial $\mathrm{GaN}$ through the schematic diagram, and further explained the effectiveness of our method. Our work provides a pathway for GaN epitaxy on the h-BN surface, which improves its surface morphology and crystal quality. Furthermore, high-quality III-nitride electronic devices and optoelectronic devices based on two-dimensional materials can be obtained through this method.

Author Contributions: Conceptualization, P.L. and S.X.; data curation, J.W. (Jinxing Wu), H.T., and X.Z.; writing-review and editing, J.W. (Jinxing Wu) and S.X.; investigation, J.W. (Jiangtao Wu), Y.W., and W.Y.; validation, Y.Z. and Y.H. All authors have read and agreed to the published version of the manuscript.

Funding: This research was funded by the National Key R\&D Program of China (Grant No. 2016YFB0400800), the National Natural Science Foundation of China (Grant No. 61634005), and the Key R\&D projects of Shaanxi Province (Grant Nos. 2018ZDCXL-GY-01-07 and 2018ZDCXL-GY-01-02-02).

Conflicts of Interest: The authors declare no conflict of interest.

\section{References}

1. Kolbe, T.; Knauer, A.; Rass, J.; Cho, H.K.; Hagedorn, S.; Einfeldt, S.; Weyers, M. Effect of electron blocking layer doping and composition on the performance of $310 \mathrm{~nm}$ light emitting diodes. Materials 2017, 10, 1396. [CrossRef] [PubMed]

2. Kim, H.S.; Kang, M.J.; Kim, J.J.; Seo, K.S.; Cha, H.Y. Effects of Recessed-Gate Structure on AlGaN/GaN-on-SiC MIS-HEMTs with Thin $\mathrm{AlO}_{\mathrm{x}} \mathrm{N}_{\mathrm{y}}$ MIS Gate. Materials 2020, 13, 1538. [CrossRef] [PubMed]

3. Wang, Y.; Li, P.; Zhang, X.; Xu, S.; Zhou, X.; Wu, J.; Hao, Y. Using a Multi-Layer Stacked AlGaN/GaN Structure to Improve the Current Spreading Performance of Ultraviolet Light-Emitting Diodes. Materials 2020, 13, 454. [CrossRef] [PubMed]

4. Al Balushi, Z.Y.; Wang, K.; Ghosh, R.K.; Vilá, R.A.; Eichfeld, S.M.; Caldwell, J.D.; Qin, X.Y.; Lin, Y.C.; DeSario, P.A.; Stone, G.; et al. Two-dimensional gallium nitride realized via graphene encapsulation. Nat. Mater. 2016, 15, 1166-1171. [CrossRef]

5. Tao, H.; Xu, S.; Zhang, J.; Li, P.; Lin, Z.; Hao, Y. Numerical investigation on the enhanced performance of N-polar AlGaN-based ultraviolet light-emitting diodes with superlattice p-type doping. IEEE Trans. Electron Devices 2018, 66, 478-484. [CrossRef]

6. Peng, R.; Meng, X.; Xu, S.; Zhang, J.; Li, P.; Huang, J.; Hao, Y. Study on Dislocation Annihilation Mechanism of the High-Quality GaN Grown on Sputtered AlN/PSS and Its Application in Green Light-Emitting Diodes. IEEE Trans. Electron Devices 2019, 66, 2243-2248. [CrossRef]

7. Roccaforte, F.; Greco, G.; Fiorenza, P.; Iucolano, F. An overview of normally-off GaN-based high electron mobility transistors. Materials 2019, 12, 1599. [CrossRef]

8. Bour, D.P.; Nickel, N.M.; Van de Walle, C.G.; Kneissl, M.S.; Krusor, B.S.; Mei, P.; Johnson, N.M. Polycrystalline nitride semiconductor light-emitting diodes fabricated on quartz substrates. Appl. Phys. Lett. 2000, 76, 2182-2184. [CrossRef]

9. Kelly, M.K.; Vaudo, R.P.; Phanse, V.M.; Görgens, L.; Ambacher, O.; Stutzmann, M. Large free-standing GaN substrates by hydride vapor phase epitaxy and laser-induced liftoff. Jpn. J. Appl. Phys. 1999, 38, L217-L219. [CrossRef]

10. Rogers, D.J.; Teherani, F.H.; Ougazzaden, A.; Gautier, S.; Divay, L.; Lusson, A.; Correira, M.R. Use of $\mathrm{ZnO}$ thin films as sacrificial templates for metal organic vapor phase epitaxy and chemical lift-off of GaN. Appl. Phys. Lett. 2007, 91, 071120. [CrossRef] 
11. Yu, J.; Wang, L.; Hao, Z.; Luo, Y.; Sun, C.; Wang, J.; Li, H. Van der Waals Epitaxy of III-Nitride Semiconductors Based on 2D Materials for Flexible Applications. Adv. Mater. 2020, 32, 1903407. [CrossRef] [PubMed]

12. Jia, Y.; Ning, J.; Zhang, J.; Yan, C.; Wang, B.; Zhang, Y.; Hao, Y. Transferable GaN Enabled by Selective Nucleation of AlN on Graphene for High-Brightness Violet Light-Emitting Diodes. Adv. Opt. Mater. 2020, 8, 1901632. [CrossRef]

13. Chang, H.; Chen, Z.; Li, W.; Yan, J.; Hou, R.; Yang, S.; Liu, Z.; Yuan, G.; Wang, J.; Li, J.; et al. Graphene-assisted quasi-van der Waals epitaxy of AlN film for ultraviolet light emitting diodes on nano-patterned sapphire substrate. Appl. Phys. Lett. 2019, 114, 091107. [CrossRef]

14. Paduano, Q.; Snure, M.; Siegel, G.; Thomson, D.; Look, D. Growth and characteristics of AlGaN/GaN heterostructures on $\mathrm{sp}^{2}$-bonded $\mathrm{BN}$ by metal-organic chemical vapor deposition. J. Mater. Res. 2016, 31, 2204-2213. [CrossRef]

15. Ayari, T.; Sundaram, S.; Li, X.; El Gmili, Y.; Voss, P.L.; Salvestrini, J.P.; Ougazzaden, A. Wafer-scale controlled exfoliation of metal organic vapor phase epitaxy grown InGaN/GaN multi quantum well structures using low-tack two-dimensional layered h-BN. Appl. Phys. Lett. 2016, 108, 171106. [CrossRef]

16. Kim, J.; Bayram, C.; Park, H.; Cheng, C.W.; Dimitrakopoulos, C.; Ott, J.A.; Reuter, K.B.; Bedell, S.W.; Sadana, D.K. Principle of direct van der Waals epitaxy of single-crystalline films on epitaxial graphene. Nat. Commun. 2014, 5, 4836. [CrossRef]

17. Choi, J.H.; Kim, J.; Yoo, H.; Liu, J.; Kim, S.; Baik, C.W.; Cho, C.R.; Kang, J.G.; Kim, M.; Braun, P.V.; et al. Heteroepitaxial Growth of GaN on Unconventional Templates and Layer-Transfer Techniques for Large-Area, Flexible/Stretchable Light-Emitting Diodes. Adv. Opt. Mater. 2016, 4, 505-521. [CrossRef]

18. Freitas, R.R.Q.; Gueorguiev, G.K.; de Brito Mota, F.; De Castilho, C.M.C.; Stafström, S. Kakanakova-Georgieva, A. Reactivity of adducts relevant to the deposition of hexagonal BN from first-principles calculations. Chem. Phys. Lett. 2013, 583, 119-124. [CrossRef]

19. Dos Santos, R.B.; de Brito Mota, F.; Rivelino, R.; Kakanakova-Georgieva, A.; Gueorguiev, G.K. Van der Waals stacks of few-layer h-AlN with graphene: An ab initio study of structural, interaction and electronic properties. Nanotechnology 2016, 27, 145601. [CrossRef]

20. Hiroki, M.; Kumakura, K.; Kobayashi, Y.; Akasaka, T.; Makimoto, T.; Yamamoto, H. Suppression of self-heating effect in AlGaN/GaN high electron mobility transistors by substrate-transfer technology using h-BN. Appl. Phys. Lett. 2014, 105, 193509. [CrossRef]

21. Gupta, P.; Rahman, A.A.; Hatui, N.; Parmar, J.B.; Chalke, B.A.; Bapat, R.D.; Purandare, S.C.; Deshmukh, M.M.; Bhattacharya, A. Free-standing semipolar III-nitride quantum well structures grown on chemical vapor deposited graphene layers. Appl. Phys. Lett. 2013, 103, 181108. [CrossRef]

22. Kobayashi, Y.; Kumakura, K.; Akasaka, T.; Makimoto, T. Layered boron nitride as a release layer for mechanical transfer of GaN-based devices. Nature 2012, 484, 223-227. [CrossRef] [PubMed]

23. Wu, Q.; Yan, J.; Zhang, L.; Chen, X.; Wei, T.; Li, Y.; Liu, Z.; Wei, X.; Zhang, Y.; Wang, J.; et al. Growth mechanism of AlN on hexagonal BN/sapphire substrate by metal-organic chemical vapor deposition. CrystEngComm 2017, 19, 5849-5856. [CrossRef]

24. Wu, Q.; Guo, Y.; Sundaram, S.; Yan, J.; Zhang, L.; Wei, T.; Wei, X.; Wang, J.; Ougazzaden, A.; Li, J. Exfoliation of AlN film using two-dimensional multilayer hexagonal BN for deep-ultraviolet light-emitting diodes. Appl. Phys. Express 2019, 12, 015505. [CrossRef]

25. Chen, Z.; Liu, Z.; Wei, T.; Yang, S.; Dou, Z.; Wang, Y.; Ci, H.; Chang, H.; Qi, Y.; Yan, J.; et al. Improved epitaxy of AlN film for deep-ultraviolet light-emitting diodes enabled by graphene. Adv. Mater. 2019, 31, 1807345. [CrossRef] [PubMed]

26. Amano, H.; Sawaki, N.; Akasaki, I.; Toyoda, Y. Metalorganic vapor phase epitaxial growth of a high quality GaN film using an AlN buffer layer. Appl. Phys. Lett. 1986, 48, 353-355. [CrossRef]

27. Kong, W.; Jiao, W.Y.; Li, J.C.; Collar, K.; Kim, T.H.; Leach, J.H.; Brown, A.S. Effect of strain in sputtered AlN buffer layers on the growth of GaN by molecular beam epitaxy. Appl. Phys. Lett. 2015, 107, 032102. [CrossRef]

28. Paskova, T.; Valcheva, E.; Birch, J.; Tungasmita, S.; Persson, P.O.; Paskov, P.P.; Monemar, B. Defect and stress relaxation in HVPE-GaN films using high temperature reactively sputtered AlN buffer. J. Cryst. Growth 2001, 230, 381-386. [CrossRef] 
29. Dos Santos, R.B.; Rivelino, R.; Mota, F.D.; Gueorguiev, G.K.; Kakanakova-Georgieva, A. Dopant species with $\mathrm{Al}-\mathrm{Si}$ and N-Si bonding in the MOCVD of AlN implementing trimethylaluminum, ammonia and silane. J. Phys. D-Appl. Phys. 2015, 48, 295104. [CrossRef]

30. Zhang, J.; Tian, W.; Wu, F.; Wan, Q.; Wang, Z.; Zhang, J.; Li, Y.; Dai, J.; Fang, Y.; Wu, Z.; et al. The effects of substrate nitridation on the growth of nonpolar a-plane $\mathrm{GaN}$ on r-plane sapphire by metalorganic chemical vapor deposition. Appl. Surf. Sci. 2014, 307, 525-532. [CrossRef]

31. Davydov, V.Y.; Kitaev, Y.E.; Goncharuk, I.N.; Smirnov, A.N.; Graul, J.; Semchinova, O.; Uffmann, D.; Smirnov, M.; Mirgorodsky, A.P.; Evarestov, R.A. Phonon dispersion and Raman scattering in hexagonal GaN and AlN. Phys. Rev. B 1998, 58, 12899-12907. [CrossRef]

32. Jiang, T.; Xu, S.R.; Zhang, J.C.; Xie, Y.; Hao, Y. Spatially resolved and orientation dependent Raman mapping of epitaxial lateral overgrowth nonpolar a-plane GaN on r-plane sapphire. Sci. Rep. 2016, 6, 19955. [CrossRef] [PubMed]

33. Kitamura, T.; Nakashima, S.; Nakamura, N.; Furuta, K.; Okumura, H. Raman scattering analysis of gan with various dislocation densities. Phys. Status Solidi(c) 2010, 5, 1789-1791. [CrossRef]

34. Niikura, E.; Murakawa, K.; Hasegawa, F.; Kawanishi, H. Improvement of crystal quality of AlN and AlGaN epitaxial layers by controlling the strain with the (AlN/GaN) multi-buffer layer. J. Cryst. Growth 2007, 298, 345-348. [CrossRef]

35. Heinke, H.; Kirchner, V.; Einfeldt, S.; Hommel, D. X-ray diffraction analysis of the defect structure in epitaxial gan. Appl. Phys. Lett. 2000, 77, 2145-2147. [CrossRef]

Publisher's Note: MDPI stays neutral with regard to jurisdictional claims in published maps and institutional affiliations.

(C) 2020 by the authors. Licensee MDPI, Basel, Switzerland. This article is an open access article distributed under the terms and conditions of the Creative Commons Attribution (CC BY) license (http://creativecommons.org/licenses/by/4.0/). 